\title{
A Mobile Service Assisted Procedural Assessment Method-Taking the Teaching of Linear Algebra as an Example
}

\author{
Xiaopin Zhong ${ }^{1, \mathrm{a},}$
}

\author{
${ }^{l}$ College of Mechatronics and Control Engineering, Shenzhen University, Shenzhen, Guangdong, China \\ axzhong@szu.edu.cn \\ *corresponding author
}

\begin{abstract}
The traditional procedural assessment method lacks operability and can not give full play to the advantages of procedural assessment. In this paper, a process teaching assessment method based on mobile service is proposed, which uses Internet tools to implement a multi-functional online testing and performance statistics assistant system by means of remote mobile service. The teaching method of using this system can effectively improve the teaching quality, lighten the burden of teachers' procedural evaluation, improve the fairness of assessment, and cultivate students' habit of concentrating in class.
\end{abstract}

Keywords: procedural assessment, mobile service, linear algebra

\section{INTRODUCTION}

The assessment model of students' performance has always been a controversial point in classroom teaching. Most undergraduate courses adopt the combination of final examination and procedural assessment. They are mainly divided into two types: final examination-oriented and process assessment-oriented.

Among them, procedural assessment increases the proportion of normal assessment, which is considered to reflect the teaching effect more flexibly and comprehensively. Constructing procedural evaluation and multiple assessment methods is the key area of university teaching research and reform. In the author's opinion, although many studies have put forward process-oriented teaching effectiveness evaluation methods $[1,2]$, most of the current non-practical classroom teaching process assessment lacks maneuverability, which is manifested in at least but not limited to the following important aspects.

First, university teachers have limited familiarity with students. Each course usually has only 2-4 hours per week, and teaching time is limited, not to mention that university teachers are also charged with many classes, different courses of teaching and many scientific research tasks. Teachers are unlikely to be able to acquaint all students with [3-5]. This will inevitably affect the reasonableness and fairness of the assessment in peacetime.

Second, there are few alternative examination methods such as questions, classroom exercises and so on, and the defects are obvious [6]. Because of the limited time, the scope of the examination objects is very narrow, only a few students are assessed, and the degree of difficulty of the examination questions varies greatly, and the examination questions are forgotten after the examination. Classroom exercises use the same examination questions, and the students at the next table copy each other also affect the fairness of the evaluation.

Third, students' classroom performance lacks scientific criteria. Usually, the assessment depends more on the subjective judgment of the teachers on the students' performance.

Fourth, the usual assessment usually includes attendance, but the traditional way of checking attendance by name is time-consuming and laborious. According to experience, checking name of a 50 people's class takes about five minutes. This kind of checking attendance will affect the fluency of classroom teaching, so the number of checking times is very limited.

Fifth, the task of correcting classroom tests after class is arduous, and the statistics of results are time-consuming and laborious. At present, most of the methods used can not achieve automation. Teachers' energy is limited. Therefore the process assessment greatly restricts teachers' scientific research work and innovation.

To sum up, the traditional process assessment method has many congenital defects and can not give full play to the advantages of the process assessment. The author believes that mobile intelligent terminal has been perfectly promoted [7-9]. This study will take the attempt of teaching reform of linear algebra as an example, and propose a procedural teaching assessment method based on mobile service. That is to say, using Internet tools and remote mobile services, we can realize a multi-functional online test and performance statistics assistant system, which can solve the drawbacks of the above-mentioned procedural assessment. This will greatly change the traditional "tasteless or meaningless" procedural assessment method and improve the teaching effect of basic courses in universities.

\section{MOBILE SERVICE BASED PROCEDURAL ASSESSMENT METHOD}

In recent years, with the popularity of platform ecology such as Wechat, mobile services have extended to all walks of life, including the education industry also has many application cases [7-9]. This paper proposes a new method of process assessment based on mobile service, with the help of 
students' mobile terminals and Wechat platforms.

\subsection{Mobile Service Scheme}

The scheme used in this paper is shown in Figure 1. In the server side, there are three functional modules that interact with the mobile side, as shown in the green box, and several hidden modules that implement various functions, as shown in the gray box.

In the $\mathrm{QR}$ code generation module, we use time stamps and other methods to encrypt the connection, so that the scan validity period is limited to about 2 seconds to 5 seconds. This can prevent students from using picture transmission to cheat. Compared with the traditional rough positioning method of GPS location, this scheme can precisely limit attendance checking in the classroom.

In the random test generation module, random question generation for in-class test is realized. An important feature of the course Linear Algebra is that it can revolve around the concepts of matrix, determinant, inversion and rank calculation which are easy to be programmed, and the steps can also be standardized. It is easy to provide the process of problem solving and to facilitate students' self-evaluation of in-class tests. Different from the traditional way of question bank extraction, this random generation model can theoretically generate unlimited kinds of questions, and completely eliminate test plagiarism among students at the next table. For a demonstration of the random generation, please refer to http://www.alors.cn/examGen/gentest.php.

In order to avoid increasing teachers' burden on checking answers of different test questions, the server integrates students' answers with machine problem-solving process and generates comparative reports, which is convenient for teachers to use debris time in mobile terminals and also for students to self-evaluate their learning situation.

In mobile front-end, only the scanner function and browser function of Wechat are needed, and complex front-end development is not needed, which can greatly save the cost of implementation.

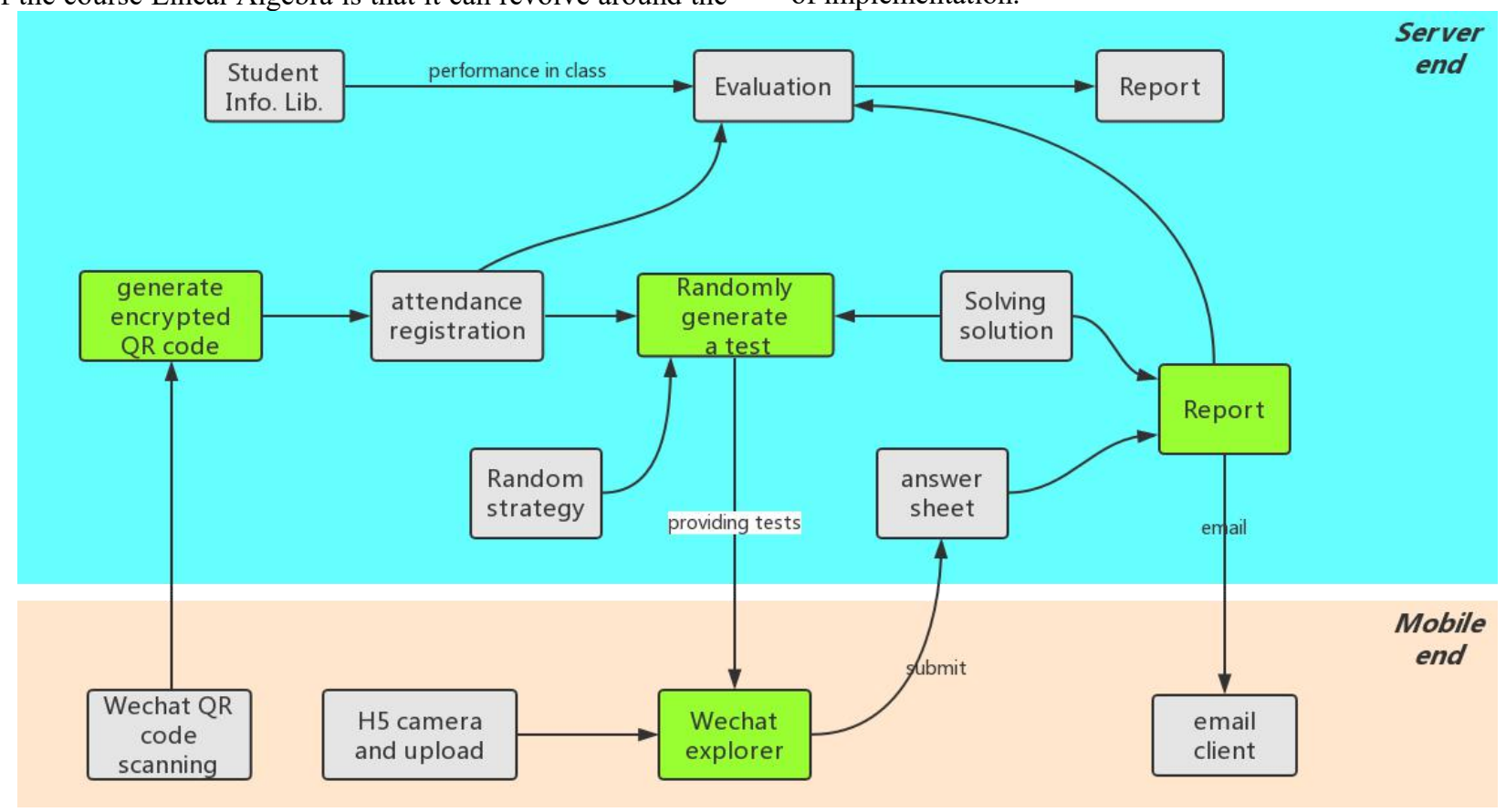

Figure 1 The schematic diagram of the implementation scheme of the procedural assessment system proposed

\subsection{Mobile Service Assisted Procedural Assessment}

The basic process of the teaching method of mobile service assisted procedural assessment is as follows. First, the teacher puts forward scanning attendance and automatically registers at the end of code scanning. Secondly, in the course of teaching, when appropriate, the request for in-class testing is put forward. The content of testing is selected according to the progress of the chapters. And the system randomly generates test questions for each student who enters from the scanning. Then, the students complete the test and take photos to upload and submit the answer. The system forms the test report together with the answer solved by machine, and sends it to the student's email or to the front end of Wechat. Finally, the teacher can check it on the mobile terminal in the time of debris, and give the score according to the test report. At the end of each semester, the system can generate process evaluation reports for attendance and in-class testing.

\subsection{Implementation Effect}

This method has been successfully applied to some teaching work of Linear Algebra in Shenzhen University in the past three years, realizing a new method of process assessment, liberating a considerable part of teaching energy, and effectively improving the teaching effect. From the final examination results of the same major, the author used the method of random generation of final examination questions for three consecutive years $(2016,2017,2018)$ to ensure that the three-year examination papers have the same examining points and the same difficulty. In the past three years, the system has been gradually improved, and the number of 
College Teachers and Students". Heilongjiang Researches on Higher Education,2016,(02):97-100.

[6] Yasushi Michita. "Effect of Question-Asking Training in a College Lecture Class on Learners' Attitudes and Ability to Ask Questions". The Japanese Journal of Educational Psychology, 2011, 59(2):193-205.

[7] Chen Heng, Li Hong-da, Zhao Xiao-yann. "Design and Implementation of University Class Attendance System Base on WeChat". Computer Engineering and Software. 2018, 39(3):45-47.

[8] Gao Xinfeng, Chen Li, Zheng Qinhua. "Research on Out-of-class Learning Interaction Based on Wechat Group". Modern Distance Education,2018,(05):48-56.

[9] Fang Yifei. "The study on specific application of Wechat in university classroom teaching". The Science Education Articles Collects. 2016, (10):35-36.

\section{CONCLUSION}

In view of some problems in the process assessment classroom teaching in universities, this paper proposes a mobile service-assisted teaching method, and takes the classroom teaching of Linear Algebra as an example. Whether from the teaching quality data or from the students' comments, the results show that the effectiveness of the method. Therefore, it can improve the teaching quality, reduce the burden of teachers' procedural assessment, improve the fairness of assessment, and cultivate students' habit of concentrating in class. This method can be extended to the classroom teaching of some basic courses in universities.

\section{ACKNOWLEDGMENT}

This research is one of the phased achievements of a teaching reform research project of Shenzhen University in 2017, No. JG2017033.

\section{REFERENCES}

[1] Zhang Xuerong, Qiao Yiyue. "Analysis on Implementation Effect of Learning Process Assessment". Vocational and Technical Education,2018,39(14):55-59.

[2] Yin Ansheng, Yuan Zhoumin. "Study on the Comprehensive Model of the Process-oriented Teaching Effect Evaluation". Heilongjiang Researches on Higher Education,2016,(12):67-71.

[3] Wang Changhua, Jin Yu, Geng Gretchen. "On the Teacher-Student Relationships and Teachers' Nonverbal Immediacy". Journal of National Academy of Education Administration,2017,(02):63-70.

[4] Zhao Zhenjie. "Exploring the Relationship Management of Between University Faculty and Student in Current China". Teacher Education Research,2015,27(05):89-93.

[5] Wang Dengdeng, Yuan Liqing. "The Review of Ten Years' Domestic Research on the Relationship Between 